\title{
High sodium:potassium intake ratio increases the risk for all-cause mortality: the REasons for Geographic And Racial Differences in Stroke (REGARDS) study
}

\author{
Suzanne E. Judd ${ }^{1}$, Kristal J. Aaron ${ }^{2}$, Abraham J. Letter ${ }^{1}$, Paul Muntner ${ }^{3}$, Nancy S. Jenny ${ }^{4}$, \\ Ruth C. Campbell ${ }^{5}$, Edmond K. Kabagambe ${ }^{3}$, Emily B. Levitan ${ }^{3}$, Deborah A. Levine ${ }^{6}$, James M. Shikany ${ }^{7}$, \\ Monika Safford ${ }^{7}$ and Daniel T. Lackland ${ }^{5}$ \\ ${ }^{1}$ Department of Biostatistics, School of Public Health, University of Alabama at Birmingham, 1665 University Boulevard, Birmingham, AL \\ 35924-0022, USA \\ ${ }^{2}$ Department of Medicine, University of Alabama at Birmingham, Birmingham, AL, USA \\ ${ }^{3}$ Department of Epidemiology, School of Public Health, University of Alabama at Birmingham, Birmingham, AL, USA \\ ${ }^{4}$ Department of Pathology, School of Medicine, University of Vermont, Burlington, VT, USA \\ ${ }^{5}$ Department of Neurosciences, Medical University of South Carolina, Charleston, SC, USA \\ ${ }^{6}$ Department of Medicine, University of Michigan and Ann Arbor VA Health Systems, Ann Arbor, MI, USA \\ ${ }^{7}$ Division of Preventive Medicine, School of Medicine, University of Alabama at Birmingham, Birmingham, AL, USA
}

(Received 17 July 2012 - Final revision received 1 November 2012 - Accepted 30 November 2012)

Journal of Nutritional Science (2013), vol. 2, e13, page 1 of 8

doi:10.1017/jns.2013.4

Abstract

Increased dietary $\mathrm{Na}$ intake and decreased dietary $\mathrm{K}$ intake are associated with higher blood pressure. It is not known whether the dietary Na:K ratio is associated with all-cause mortality or stroke incidence and whether this relationship varies according to race. Between 2003 and 2007, the REasons for Geographic And Racial Differences in Stroke (REGARDS) cohort enrolled 30239 black and white Americans aged 45 years or older. Diet was assessed using the Block 98 FFQ and was available on 21374 participants. The Na:K ratio was modelled in race- and sex-specific quintiles for all analyses, with the lowest quintile (Q1) as the reference group. Data on other covariates were collected using both an in-home assessment and telephone interviews. We identified 1779 deaths and 363 strokes over a mean of 4.9 years. We used Cox proportional hazards models to obtain multivariable-adjusted hazard ratios (HR). In the highest quintile (Q5), a high Na:K ratio was associated with all-cause mortality (Q5 v. Q1 for whites: HR 1.22; $95 \%$ CI 1.00, 1.47 , $P$ for trend $=0.084$; for blacks: HR 1.36; $95 \%$ CI 1.04, 1.77, P for trend $=0 \cdot 028$ ). A high Na:K ratio was not significantly associated with stroke in whites (HR 1.29; $95 \%$ CI $0.88,1.90$ ) or blacks (HR 1.39; $95 \%$ CI $0.78,2 \cdot 48$ ), partly because of the low number of stroke events. In the REGARDS study, a high $\mathrm{Na}: \mathrm{K}$ ratio was associated with all-cause mortality and there was a suggestive association between the Na:K ratio and stroke. These data support the policies targeted at reduction of $\mathrm{Na}$ from the food supply and recommendations to increase $\mathrm{K}$ intake.

Key words: Stroke: Death: Race: Sodium

Introduction

Since the turn of the twentieth century, reducing $\mathrm{Na}$ intake has been a key target for the prevention of hypertension, a major risk factor for stroke ${ }^{(1,2)}$. Although $\mathrm{Na}$ occurs naturally in food, the excess $\mathrm{Na}$ ingested in Western cultures comes largely from processed food. Reduction of dietary $\mathrm{Na}$ is once again

* Corresponding author: Dr S. E. Judd, email sejudd@uab.edu

Abbreviations: HR, hazard ratio; REGARDS, REasons for Geographic And Racial Differences in Stroke.

(C) The Author(s) 2013. The online version of this article is published within an Open Access environment subject to the conditions of the Creative Commons Attribution-NonCommercial-ShareAlike licence <http://creativecommons.org/licenses/by-nc-sa/2.5/>. The written permission of 
gaining momentum with new recommendations from the United States Department of Agriculture ${ }^{(3)}$ and state organisations introducing $\mathrm{Na}$ reduction campaigns ${ }^{(4,5)}$. Despite abundant research suggesting that lower dietary $\mathrm{Na}$ would decrease hypertension and stroke ${ }^{(6)}, \mathrm{Na}$ intake in the USA remains well above recommended levels, with only $5.5 \%$ of Americans meeting United States Department of Agriculture targets ${ }^{(7)}$. Also, dietary $\mathrm{Na}$ may contribute to the higher rates of stroke and stroke mortality observed in black Americans compared with white Americans ${ }^{(8)}$.

In contrast with $\mathrm{Na}$, increased dietary $\mathrm{K}$ may reduce the burden of hypertension and stroke. Increased $\mathrm{K}$ has been shown to lower blood pressure and this beneficial effect may be greater in the setting of a high-Na diet ${ }^{(9-12)}$. In fact, US veterans switching from regular salt to a K-enriched salt experienced reduced CVD mortality ${ }^{(13)}$. Dietary $\mathrm{K}$ may also contribute to racial differences in stroke and stroke mortality because black Americans have a lower $\mathrm{K}$ intake than white Americans ${ }^{(14)}$.

Taken together, the dietary $\mathrm{Na}: \mathrm{K}$ ratio may explain demographic differences in stroke and stroke mortality in the USA. Using the ratio provides a means of balancing the detrimental effects of $\mathrm{Na}$ and beneficial effects of $\mathrm{K}$ in one measure. Recent analyses using mortality follow-up from the National Health and Nutrition Examination Survey demonstrated that the dietary $\mathrm{Na}: \mathrm{K}$ ratio was more strongly associated with CVD mortality than $\mathrm{Na}$ alone, but lacked power to examine this association by race ${ }^{(15)}$. Very little is known about the question whether the dietary $\mathrm{Na}: \mathrm{K}$ ratio is associated with all-cause mortality and stroke incidence and whether these relationships differ across racial groups.

The primary hypothesis of this study was that an increased dietary $\mathrm{Na}: \mathrm{K}$ ratio is associated with greater risk of death and incident stroke and that this association will be stronger in blacks than in whites. To test this hypothesis, we utilised the data from participants of the REasons for Geographic And Racial Differences in Stroke (REGARDS) study, a large population-based prospective cohort study of black and white US adults aged 45 years or older ${ }^{(16)}$.

\section{Experimental methods}

\section{Participants}

The design and objectives of the REGARDS study have been described previously ${ }^{(16)}$. By design, the study oversampled blacks and included an approximately equal representation of men and women. Enrolment began in January 2003 and ended in October 2007. Approximately half of the participants were recruited from the 'Stroke Belt' (states of North Carolina, South Carolina, Georgia, Tennessee, Alabama, Mississippi, Arkansas and Louisiana). The REGARDS study participants were identified using a commercial, nation-wide list of over 250 million individuals in the USA (Genesys Incorporated). A trained interviewer contacted households by telephone, and one resident per household aged 45 years or older was randomly screened for eligibility. The response rate (the percentage agreeing to be interviewed among known eligible candidates contacted after adjustment for those of unknown eligibility) was $41 \%$, which is similar to other research studies that initially contacted participants by telephone ${ }^{(17,18)}$. Exclusion criteria were: race other than black or white; active treatment for cancer; medical conditions preventing long-term participation; cognitive impairment as subjectively judged by the telephone interviewer; staying in, or on a waiting list for, a nursing home; and inability to communicate in English. This study was conducted according to the guidelines laid down in the Declaration of Helsinki and all procedures involving human subjects/patients were approved by the Institutional Review Boards at the University of Alabama Birmingham. Written informed consent was obtained from all subjects/patients.

\section{Data collection and covariates}

Data were collected during a telephone interview, an in-home visit and via self-administered questionnaires that were left for participants to complete following the in-home visit. Of relevance to the present analysis, the telephone interview included information on age, race, sex, education, household income, current smoking status and a prior diagnosis of hypertension, diabetes mellitus and myocardial infarction. During the in-home study visit, weight, height and waist circumference were measured by following a standardised protocol. BMI was calculated as weight in kilograms divided by height in metres squared. Using the mean of two blood pressure measurements, hypertension was defined as a systolic blood pressure $\geq 140 \mathrm{mmHg}$, diastolic blood pressure $\geq 90 \mathrm{mmHg}$ or selfreported use of anti-hypertensive medication. Participants were asked to fast for $10-12 \mathrm{~h}$ prior to the in-home study visit. A blood sample was collected, centrifuged, refrigerated and shipped overnight to the REGARDS central laboratory at the University of Vermont. Total cholesterol, HDLcholesterol, TAG, serum glucose and serum creatinine were measured by colorimetric reflectance spectrophotometry using the Ortho Vitros Clinical Chemistry System 950IRC instrument (Johnson \& Johnson Clinical Diagnostics) and LDL-cholesterol was calculated by the Friedewald equation for individuals with TAG $<10 \mathrm{mmol} / 1$. Dyslipidaemia was defined as a total cholesterol $\geq 6 \cdot 2 \mathrm{mmol} / 1$, LDL-cholesterol $\geq 4.1 \mathrm{mmol} / 1$, HDL-cholesterol $<1.0 \mathrm{mmol} / 1$ for men or $<1.3 \mathrm{mmol} / 1$ for women, or lipid-lowering medication use. Diabetes mellitus was defined as fasting glucose $\geq 7.0$ $\mathrm{mmol} / \mathrm{l}, \geq 11.0 \mathrm{mmol} / \mathrm{l}$ for participants who did not fast, or self-reported current use of anti-diabetes medication.

\section{Nutritional assessment}

At the end of the in-home study visit, participants were asked to complete additional questionnaires which included the Block 98 FFQ (www.Nutritionquest.com). The Block FFQ has been validated for most nutrients, including $\mathrm{Na}$ and $\mathrm{K}$, using multiple diet records ${ }^{(19)}$. Different versions of this questionnaire have been studied extensively and validated in diverse populations $^{(20)}$. The Block 98 version developed by Block Dietary Data Systems and distributed by NutritionQuest is 
an eight-page paper-and-pencil form with more than 150 multiple-choice questions based on 107 food items that can be completed in about 30-40 min. In the REGARDS study, the FFQ was self-administered and participants were asked to report on diet over the past year. Pictures were provided to help identify portion sizes. FFQ, compared with diary techniques, tend to underestimate micronutrient intake and overestimate total energy intake; however, this measurement error is usually uniformly distributed across study subpopulations ${ }^{(21,22)}$. Therefore, it is generally recommended that estimated nutrient intakes from FFQ should be reported and analysed as either ranked values (i.e. quintiles, etc.) or ratios of two different nutrients rather than absolute values per individual $^{(22)}$. Thus, race- and sex-specific quintiles of dietary $\mathrm{Na}$ $(\mathrm{mg})$ and the Na:K ratio were used in all analyses.

\section{Outcome assessment for incident stroke and death}

Every 6 months, the REGARDS study ascertains all hospitalisations by participant or proxy interview using methods described elsewhere ${ }^{(23)}$. Personnel specifically pursue medical records for all hospitalisations related to stroke, transient ischaemic attack, stroke symptoms and deaths. Medical records for strokes that occurred before baseline are not pursued. For all participants who have died, study personnel interview the next of kin or other proxy to probe for information relating to the death, inquiring if a stroke not previously reported antedated the death; any hospitalisations prior to death are collected. Once the records are received, they are first reviewed by a trained neurological nurse to verify that the record is complete, and then the information is forwarded to a team of stroke neurologists for review. In the case of deaths, the interview with next of kin or proxy and death certificate or National Death Index data are included. A stroke was defined as focal neurological symptoms lasting greater than $24 \mathrm{~h}$ or imaging positive for a new stroke. For this analysis, adjudicated stroke events were available to 1 February 2011 and we included only incident strokes. The REGARDS study is notified of a participant's death by proxies either through the mail or on the telephone during the routine 6-month telephone calls and also uses the Social Security Death Index to look for participants who may have died and for whom we do not have a proxy report of death. For this analysis, death events were available to 1 April 2011. We used all-cause mortality as an outcome in addition to adjudicated stroke.

\section{Statistical analysis}

As the association of dietary $\mathrm{Na}$ with CVD has been reported to differ by race, we decided a priori to stratify analyses for black and white participants. The distribution of participant characteristics was determined for blacks and whites separately. The prevalence and multivariable-adjusted hazard ratios (HR) for incident stroke associated with race- and sex-specific quintiles of the Na:K ratio were calculated using Cox proportional hazards models. Lastly, the prevalence and multivariable-adjusted HR for all-cause mortality were calculated by sex-specific quintile of the $\mathrm{Na}: \mathrm{K}$ ratio. Multivariable-adjusted HR were calculated in three steps. In the first step we adjusted for age, race, sex and total energy intake. In the second step we additionally adjusted for BMI, hypertension, diabetes, dyslipidaemia, history of heart disease, smoking, income and education. In the third step, which was only for analyses with stroke as an outcome, we further adjusted for bias in medical record retrieval and extraction ${ }^{(24)}$. For the mortality analysis, we performed a sensitivity analysis excluding those who died within 6 months of the baseline visit (as a proxy for terminal illness) and all participants who reported a history of stroke at baseline. As a secondary analysis, the multivariable-adjusted HR for incident stroke and all-cause mortality associated with quintiles of $\mathrm{Na}$ were calculated. Additionally, we considered the $\mathrm{Na}: \mathrm{K}$ ratio as a continuous variable for secondary analyses and examined the HR associated with $1 \mathrm{SD}$ increase in the $\mathrm{Na}: \mathrm{K}$ ratio. Analyses were performed with SAS version 9.2 software (SAS Institute, Inc.).

\section{Results}

\section{Participant characteristics}

The REGARDS cohort enrolled 30239 participants. We excluded 8603 participants because they were missing FFQ data $(n$ 6000) or answered less than $85 \%$ of the FFQ $(n$ 1557) or who had extreme energy intakes $(<3347.2 \mathrm{~kJ}$ or $>20920 \mathrm{~kJ} / \mathrm{d}$ for males $(n 689)$ and $<2092 \mathrm{~kJ}$ or $>18828$ $\mathrm{kJ} / \mathrm{d}$ for females $(n$ 357) $(4 \cdot 184 \mathrm{~kJ}=1 \mathrm{kcal}))$. Compared with blacks $(58 \%)$, whites $(81 \%)$ were more likely to return the FFQ $(P<0.001)$. College graduates $(77 \%)$ were also more likely than non-graduates $(55 \%)$ to return the FFQ $(P<0 \cdot 001)$. Participants who did not return the FFQ had a slightly higher BMI (30.0 (SD 6.4) v. 29.1 (SD 3.1) kg/m 2 , P< 0.001). Age was not associated with the likelihood of returning the FFQ.

The median $\mathrm{Na}$ intake was higher in blacks (median $=0.92$; interquartile range $=0.43$ ) than in whites (median $=0.83$; interquartile range $=0 \cdot 32$ ). Regardless of race, participants with higher dietary Na:K ratios were more likely to be hypertensives, younger, current smokers, obese and to reside in the Southeast (Table 1). Median energy intake increased across quintiles of $\mathrm{Na}: \mathrm{K}$ ratio as did the total $\mathrm{Na}$ intake.

Results on risk of death associated with increasing dietary sodium:potassium ratio

Compared with participants in the lowest quintile of $\mathrm{Na}: \mathrm{K}$ intake, those in the highest quintile were at increased risk of death (Table 2). In age-, race-, sex- and energy-adjusted models, the HR for death comparing quintile 5 (Q5) to quintile 1 (Q1) of dietary Na:K intake was 1.52 (95\% CI 1.31, 1.76). Adjustment for BMI, hypertension, diabetes, heart disease, smoking, income and education attenuated the association (HR 1.26; $95 \%$ CI 1.08, 1.47). We also examined the dietary $\mathrm{Na}: \mathrm{K}$ ratio as a continuous variable. For each standard deviation $(0.28 \mathrm{mg} \mathrm{Na}$ per $\mathrm{mg} \mathrm{K})$ increase in the dietary 
Table 1. Characteristics of participants by race and quintile $(Q)$ of sodium:potassium ratio in the REasons for Geographic And Racial Differences in Stroke (REGARDS) study*

(Number of participants, percentages, medians, interquartile ranges (IQR) and ranges)

\begin{tabular}{|c|c|c|c|c|c|c|c|c|c|c|}
\hline & \multicolumn{2}{|c|}{ Q1 } & \multicolumn{2}{|c|}{ Q2 } & \multicolumn{2}{|c|}{ Q3 } & \multicolumn{2}{|c|}{ Q4 } & \multicolumn{2}{|c|}{ Q5 } \\
\hline & $n$ & $\%$ & $n$ & $\%$ & $n$ & $\%$ & $n$ & $\%$ & $n$ & $\%$ \\
\hline \multicolumn{11}{|l|}{ Black participants } \\
\hline$n$ & \multicolumn{2}{|c|}{1429} & \multicolumn{2}{|c|}{1431} & \multicolumn{2}{|c|}{1430} & \multicolumn{2}{|c|}{1431} & \multicolumn{2}{|c|}{1430} \\
\hline \multicolumn{11}{|l|}{ Na:K ratio } \\
\hline Median & \multicolumn{2}{|c|}{0.56} & \multicolumn{2}{|c|}{0.76} & \multicolumn{2}{|c|}{0.92} & \multicolumn{2}{|c|}{1.09} & \multicolumn{2}{|c|}{$1 \cdot 36$} \\
\hline IQR & \multicolumn{2}{|c|}{0.13} & \multicolumn{2}{|c|}{0.09} & \multicolumn{2}{|c|}{0.09} & \multicolumn{2}{|c|}{0.10} & \multicolumn{2}{|c|}{0.23} \\
\hline Range (men) & 0.2 & & & & 0.5 & & 1.0 & & & \\
\hline Range (women) & 0.1 & & & & $0 . \varepsilon$ & & 0.9 & & & \\
\hline $\mathrm{Na}$ intake $(\mathrm{mg} / \mathrm{d})$ & & & & & & & & & & \\
\hline Median & & & & & & & & & & \\
\hline IQR & & & & & & & & & & \\
\hline Energy intake (kJ/d) & & & & & & & & & & \\
\hline Median & & & & & & & & & & \\
\hline IQR & & & & & & & & & & \\
\hline Age 65 years and older & 726 & 51 & 677 & 47 & 626 & 44 & 579 & 40 & 539 & 38 \\
\hline Southeast & 708 & 50 & 695 & 49 & 755 & 53 & 761 & 53 & 796 & 56 \\
\hline Women & 944 & 66 & 945 & 66 & 944 & 66 & 945 & 66 & 944 & 66 \\
\hline Obese & 643 & 45 & 666 & 47 & 715 & 50 & 716 & 50 & 731 & 51 \\
\hline History of stroke & 104 & $7 \cdot 3$ & 83 & $5 \cdot 8$ & 92 & $6 \cdot 4$ & 94 & $6 \cdot 6$ & 96 & $6 \cdot 7$ \\
\hline History of CAD & 213 & 15 & 201 & 14 & 192 & 13 & 169 & 12 & 208 & 15 \\
\hline History of HTN & 994 & 70 & 1015 & 71 & 1017 & 71 & 999 & 70 & 1043 & 73 \\
\hline Current smoker & 195 & 14 & 213 & 15 & 213 & 15 & 290 & 20 & 316 & 22 \\
\hline White participants & & & & & & & & & & \\
\hline$n$ & & & & & & & & & & \\
\hline $\mathrm{Na}: \mathrm{K}$ ratio & & & & & & & & & & \\
\hline Median & & & & & & & & & & \\
\hline IQR & & & & & & & & & & \\
\hline Range (men) & 0.2 & & & & $0 . \varepsilon$ & & 0.8 & & & \\
\hline Range (women) & $0 . c$ & & & & 0.7 & & 0.8 & & & \\
\hline $\mathrm{Na}$ intake $(\mathrm{mg} / \mathrm{d})$ & & & & & & & & & & \\
\hline Median & & & & & & & & & & \\
\hline IQR & & & & & & & & & & \\
\hline Energy intake (kJ/d) & & & & & & & & & & \\
\hline Median & & & & & & & & & & \\
\hline IQR & & & & & & & & & & \\
\hline Age 65 years and older & 1718 & 60 & 1566 & 55 & 1460 & 51 & 1428 & 50 & 1269 & 45 \\
\hline Southeast & 1529 & 54 & 1627 & 57 & 1668 & 59 & 1743 & 61 & 1749 & 61 \\
\hline Women & 1446 & 51 & 1446 & 51 & 1447 & 51 & 1446 & 51 & 1446 & 51 \\
\hline Obese & 637 & 22 & 750 & 26 & 825 & 29 & 952 & 33 & 1126 & 40 \\
\hline History of stroke & 137 & $4 \cdot 8$ & 127 & 4.5 & 137 & $4 \cdot 8$ & 126 & 4.4 & 144 & $5 \cdot 1$ \\
\hline History of CAD & 539 & 19 & 537 & 19 & 541 & 19 & 486 & 17 & 533 & 19 \\
\hline History of HTN & 1366 & 48 & 1377 & 48 & 1405 & 49 & 1439 & 51 & 1494 & 52 \\
\hline Current smoker & 246 & 8.6 & 275 & 9.7 & 341 & 12 & 360 & 13 & 459 & 16 \\
\hline
\end{tabular}

CAD, coronary artery disease; HTN, hypertension.

${ }^{*}$ Quintiles are race and sex specific.

$\mathrm{Na}: \mathrm{K}$ ratio, the risk of death increased by $11 \%$ (HR 1.11; $95 \% \mathrm{CI}=1 \cdot 05,1 \cdot 16)$. Although the $P$ value for interaction was not statistically significant, we further examined this association stratified by race based on our a priori hypothesis. The risk of each standard deviation increase in dietary $\mathrm{Na}: \mathrm{K}$ ratio was similar in blacks (HR 1.12; $95 \%$ CI 1.04, 1.21) and whites (HR 1.09; $95 \%$ CI 1.02, 1.16) (Table 2).

\section{Results on risk of incident stroke associated with increasing dietary sodium:potassium ratio}

Over a mean of 4.9 years of follow-up, we accumulated 363 stroke events. Although there was a suggestion for increased risk of stroke among individuals in Q5 compared with Q1 of dietary $\mathrm{Na}: \mathrm{K}$ ratio, this association was not statistically significant (Table 3) in analysis before or after stratifying by race. In the fully adjusted model comparing Q5 with Q1 of dietary Na:K ratio, the HR of incident stroke was $1.29(95 \%$ CI $0.94,1.78)$. For a $1 \mathrm{SD}$ increase in dietary $\mathrm{Na}: \mathrm{K}$ ratio, the HR of incident stroke was 1.08 (95\% CI 0.98, 1.20). Similar to the results for death, there was no statistically significant difference by race. In blacks, all quintiles of dietary $\mathrm{Na}: \mathrm{K}$ intake had a higher risk of incident stroke compared with Q1. This association was not linear. In whites, the association between dietary $\mathrm{Na}: \mathrm{K}$ intake and incident stroke was fairly flat across quintiles with a slight increase in Q5 v. Q1 (HR $1.29 ; 95 \%$ CI $0.88,1.90)$. In a sensitivity analysis limited to those who survived at least 6 months beyond the baseline visit and who did not have a history of stroke at baseline, the results were similar (data not shown). 
Table 2. Risk of death associated with increasing sodium:potassium ratio in the REasons for Geographic And Racial Differences in Stroke (REGARDS) study (Hazard ratios (HR) and 95\% confidence intervals)

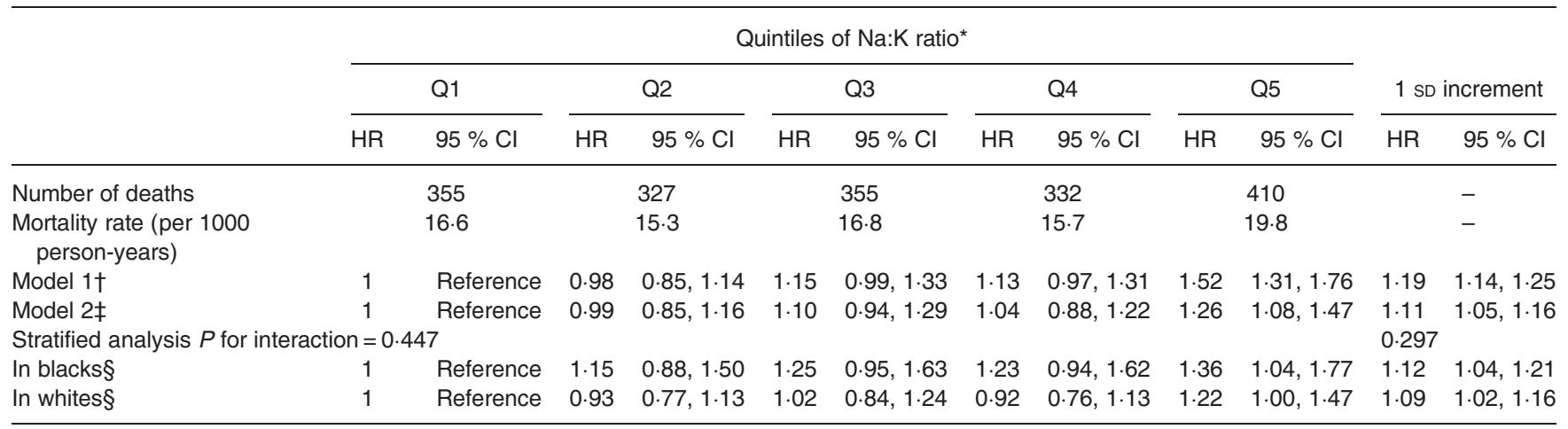

* Quintiles are race and sex specific.

† Model 1 - age, race, sex, total energy intake.

$\ddagger$ Model 2 - age, race, sex, energy, BMl, hypertension, diabetes, history of heart disease, dyslipidaemia, smoking, income, education and use of statins

$\S$ For the models stratified by race, model 2 adjustments were used and race was not included in the model.

\section{Results on risk of death and risk of incident stroke associated with increasing dietary sodium}

Increasing the dietary $\mathrm{Na}$ had a weak, non-linear association with risk of death that was attenuated after adjustment for BMI, hypertension, diabetes, dyslipidaemia, history of heart disease, smoking, income and education (Supplementary Table 1). Comparing Q5 with Q1 of dietary Na, the risk of death associated with increasing dietary $\mathrm{Na}$ was stronger for the black participants (HR 1.62; $95 \%$ CI 1.03, 2.55) than for the white participants (HR 0.94; $95 \%$ CI 0.67, 1.30). Dietary Na was associated with incident stroke in a pattern similar to that observed for the dietary Na:K ratio (Supplementary Table 2). Again, a weak non-linear association was observed. Comparing Q5 with Q1 of dietary $\mathrm{Na}$, the risk of stroke associated with increasing dietary $\mathrm{Na}$ was stronger for the black participants (HR 2.13; $95 \%$ CI $0 \cdot 88,5 \cdot 13$ ) than for the white participants
(HR 1.30; $95 \%$ CI 0.66, 2.57), though these estimates were not statistically different from one another.

\section{Food groups and dietary sodium:potassium ratio}

As we did not observe racial differences in the $\mathrm{Na}: \mathrm{K}$ ratio, we also wanted to investigate whether there were differences in food choice contributing to both high and low Na:K ratios. We used Pearson's correlation to compare grams of food from each food group with the Na:K ratio. The foods were largely similar across races. In blacks and whites, high fruit and fruit juice intakes correlated with a lower $\mathrm{Na}: \mathrm{K}$ ratio (Table 4). Processed meats, bread and non-fried chicken were each correlated with a higher $\mathrm{Na}: \mathrm{K}$ ratio. In whites, high liquor consumption was correlated with a higher $\mathrm{Na}$ :K ratio, while coffee consumption was associated with a lower

Table 3. Risk of stroke associated with increasing sodium:potassium ratio in the REasons for Geographic And Racial Differences in Stroke (REGARDS) study

(Hazard ratios (HR) and 95\% confidence intervals)

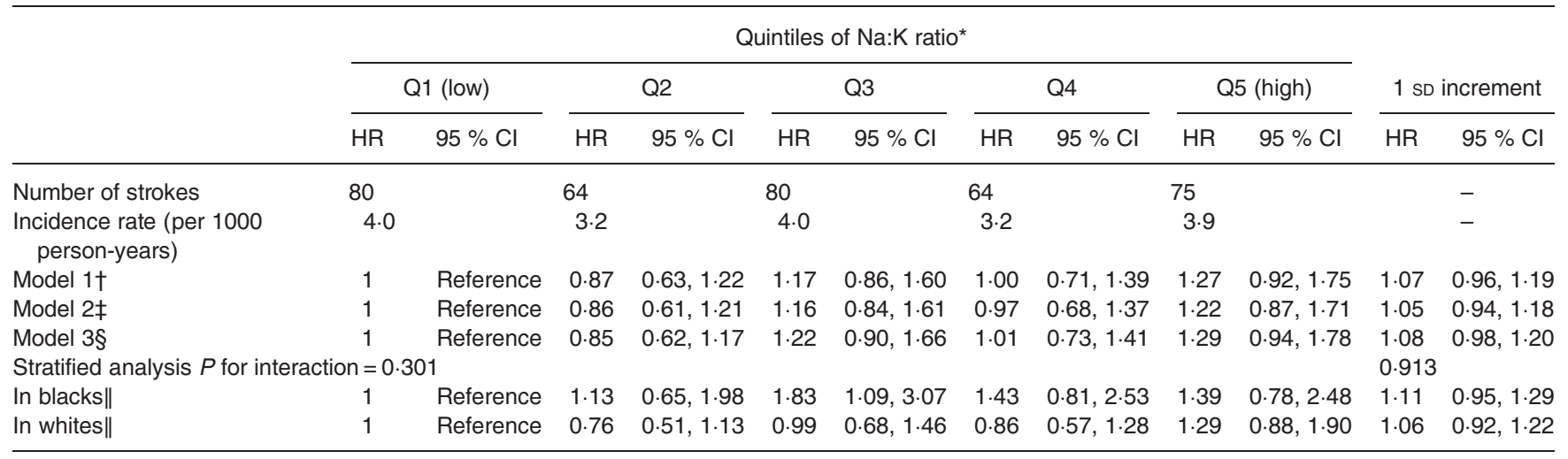

* Quintiles are race and sex specific.

† Model 1 - age, race, sex, energy.

$\ddagger$ Model 2 - age, race, sex, energy, BMI, hypertension, diabetes, history of heart disease, dyslipidaemia, smoking, income, education.

$\S$ Model 3 - age, race, sex, energy, BMI, hypertension, diabetes, history of heart disease, dyslipidaemia, smoking, income, education, bias in retrieving and extraction of medical records.

$\|$ For the models stratified by race, model 3 adjustments were used and race was not included. 
Table 4. Pearson's correlations of food groups ( $\mathrm{g} / \mathrm{d})$ with sodium: potassium ratio by race in the REasons for Geographic And Racial Differences in Stroke (REGARDS) study*

\begin{tabular}{|c|c|c|c|}
\hline \multicolumn{2}{|l|}{ Black participants } & \multicolumn{2}{|l|}{ White participants } \\
\hline Food group & $r$ & Food group & $r$ \\
\hline Non-fried chicken & 0.93 & Non-fried chicken & 0.64 \\
\hline Processed meats & 0.43 & Liquor & 0.50 \\
\hline Bread & 0.41 & Processed meats & 0.42 \\
\hline $\begin{array}{l}\text { Added fats (lard, crisco, } \\
\text { gravy) }\end{array}$ & 0.33 & Bread & 0.39 \\
\hline Fried chicken or fish & 0.28 & $\begin{array}{l}\text { Added fats (lard, crisco, } \\
\text { gravy) }\end{array}$ & 0.29 \\
\hline Low-fat milk & -0.16 & Coffee & -0.20 \\
\hline Cruciferous vegetables & -0.18 & Low-fat milk & -0.23 \\
\hline Other vegetables & -0.23 & Other vegetables & -0.25 \\
\hline Fruit juice & -0.36 & Fruit juice & -0.28 \\
\hline Fruit & -0.37 & Fruit & -0.35 \\
\hline
\end{tabular}

* For simplicity only the top five and bottom five correlations are shown. All $P<0.001$.

$\mathrm{Na}: \mathrm{K}$ ratio. In blacks, added fats such as lard and gravy were correlated with a higher $\mathrm{Na}: \mathrm{K}$ ratio, while cruciferous vegetables were associated with a lower $\mathrm{Na}: \mathrm{K}$ ratio.

\section{Discussion}

Among middle-aged and older adults, a high dietary $\mathrm{Na}: \mathrm{K}$ ratio was associated with an increased risk of death. This association was observed in blacks and whites and persisted after adjustment for several demographic, socioeconomic and other health factors. Although a similar trend was present for the risk of incident stroke, this association was not statistically significant, which most probably is due to the lower event rate for stroke compared with all-cause mortality. Our findings on higher risk of death with increasing dietary $\mathrm{Na}: \mathrm{K}$ ratio are consistent with previous research showing that a higher dietary $\mathrm{Na}: \mathrm{K}$ ratio, as measured in either urine or diet, is associated with a higher risk of death ${ }^{(25)}$. Compared with the results for dietary $\mathrm{Na}: \mathrm{K}$ ratio, the direction of the association between $\mathrm{Na}$ intake and the outcomes was similar but the magnitude of the association was weaker.

Based on previous bench and population research, we hypothesised that the association of the $\mathrm{Na}: \mathrm{K}$ ratio and both stroke and death would be higher in blacks than whites ${ }^{(26,27)}$. Salt sensitivity has been found to be highly correlated with specific phenotypic and metabolic characteristics, particularly for blacks in the USA ${ }^{(28,29)}$. In contrast with whites, salt sensitivity is prevalent in both normotensive and hypertensive blacks ${ }^{(27,30,31)}$. Diets that are high in $\mathrm{K}$ and low in $\mathrm{Na}$ (e.g. the Dietary Approaches to Stop Hypertension diet) effectively lower blood pressure in whites, but do so to a greater degree in blacks ${ }^{(32)}$. Therefore, we were surprised that we did not find racial differences in the effect of the $\mathrm{Na}: \mathrm{K}$ ratio (or $\mathrm{Na}$ alone) on stroke or mortality in this study. Inclusion of hypertension as a covariate may have dampened the observed association as it may lie in the causal pathway between higher $\mathrm{Na}$ intake and either death or stroke. Our findings suggest that an increased $\mathrm{Na}: \mathrm{K}$ ratio in the diet is harmful regardless of race.
Separating individual nutrients from overall diet quality is challenging. Therefore, it is possible that the association of a higher $\mathrm{Na}: \mathrm{K}$ ratio with increased risk of all-cause mortality that we observed is due to an improvement in overall diet quality. In fact, we observed that lower $\mathrm{Na}: \mathrm{K}$ ratio values were correlated with greater intakes of fruit, low-fat milk and vegetable intake. A lower dietary $\mathrm{Na}: \mathrm{K}$ ratio is associated with a greater intake of whole grains, low-fat dairy products, fruits, vegetables and lean meats, all of which have been associated with better health ${ }^{(33)}$. However, in our study, the association between a higher $\mathrm{Na}: \mathrm{K}$ ratio and increased risk of all-cause mortality persisted even after adjustment for alcohol, whole grain, fruit and vegetable intake. In addition to the fact that diet quality itself is associated with improved cardiovascular health, diet quality is also strongly correlated with socioeconomic status ${ }^{(34)}$. Although a possibility for residual confounding by socioeconomic status still exists, we controlled for two major indicators of socioeconomic status: income and education.

The present study has potential limitations. We are unable to assess causality due to the study's observational design. $\mathrm{Na}$ and $\mathrm{K}$ intake were estimated by assessing diet rather than multiple 24-h urine collections, which are known to more accurately reflect $\mathrm{Na}: \mathrm{K}$ intake, and we did not have the ability to capture salt added to food by the participant. Although individuals may erroneously report nutritional intake on FFQ, dietary questionnaires provide an acceptable classification of nutrient intake on a population level and are widely accepted in cohort studies with large sample sizes such as the REGARDS study ${ }^{(35,36)}$. Participants may under-report their $\mathrm{Na}$ intake and inflate their intake of more healthful foods. This potential bias would reduce the ability to detect significant associations between the Na:K ratio and outcomes in the present study. Participants who returned the FFQ were more likely to be college educated than those who did not return the FFQ. Although we controlled for education and income in our analyses, individuals of lower socioeconomic status were less represented in our study. Although the REGARDS study has a strong surveillance system, we did not have complete ascertainment of death. Despite this potential bias, we found a significant association between dietary $\mathrm{Na}: \mathrm{K}$ ratio and mortality. In addition, we did not know the cause of death and were unable to study CVD mortality. Previous studies have demonstrated stronger associations of the Na:K ratio with CVD mortality than all-cause mortality, so we would expect our findings to be strengthened with the addition of these data ${ }^{(15)}$. Despite these limitations, the REGARDS study also has several notable strengths. These strengths include the enrolment of a large sample of whites and blacks from all over continental USA, physician adjudication of strokes using medical records, and the collection of dietary data using a standardised and validated FFQ.

In the present study, individuals with a reported diet high in $\mathrm{Na}$ and low in $\mathrm{K}$ had a greater risk of death. Although the physiological mechanisms are not fully understood, diets high in $\mathrm{K}$ may mediate the adverse effects of dietary $\mathrm{Na}$, resulting in lower blood pressures and a decreased risk of stroke and stroke mortality. Data from this study reinforce recommendations to lower dietary $\mathrm{Na}$ intake and increase $\mathrm{K}$ 
intake as a means of lowering mortality in the general population, but do not support specific recommendations for different race groups. Thus, our data suggest that the implementation of strategies to reduce $\mathrm{Na}$ in the US food supply has similar implications for whites and blacks.

\section{Acknowledgements}

This research project was supported by cooperative agreement number U01 NS041588 from the National Institute of Neurological Disorders and Stroke, National Institutes of Health, Department of Health and Human Services. In addition, an investigator-initiated grant from General Mills helped pay for the cost of obtaining nutrient data from the FFQ. The authors thank the investigators and staff of the REGARDS study for their valuable contributions. A full list of participating REGARDS investigators and institutions can be found at http://www.regardsstudy.org. The content is solely the responsibility of the authors and does not necessarily represent the official views of the National Institute of Neurological Disorders and Stroke or the National Institutes of Health. E. K. K. is supported by a National Scientist Development grant (no. 0635323N) from the American Heart Association. D. A. L. received research support from the National Institutes of Health (P30 DK092926 and K23 AG040278).

\section{Supplementary material}

The supplementary material for this article can be found at http://www.journals.cambridge.org/jns

\section{References}

1. Esunge PM (1991) From blood pressure to hypertension: the history of research. J R Soc Med 84, 621.

2. Mayer J, Kline OL, Cooke JA, et al. (1969) White House Conference on Food, Nutrition, and Health. Washington, DC. http://www.nns.nih. gov/1969/full_report/White_House_Report2_S1a.pdf

3. US Department of Agriculture \& US Department of Health and Human Services (eds) (2010) Food and food components to reduce. In Dietary Guidelines for Americans, 2010, 7th ed., pp. 20 32. Washington, DC: US Government Printing Office.

4. Centers for Disease Control and Prevention (2011) Legal and policy resources on public health 'Winnable Battles'. http://www.cdc. gov/phlp/winnable/index.html (accessed 23 June 2011).

5. Appel LJ, Frohlich ED, Hall JE, et al. (2011) The importance of population-wide sodium reduction as a means to prevent cardiovascular disease and stroke: a call to action from the American Heart Association. Circulation 123, 1138-1143.

6. He J, Ogden LG, Vupputuri S, et al. (1999) Dietary sodium intake and subsequent risk of cardiovascular disease in overweight adults. JAMA 282, 2027-2034.

7. Peralez-Gunn J, Kuklina E, Keena N, et al. (2010) Sodium intake among adults - United States, 2005-2006. MMWR. Morb Mortal Wkly Rep 59, 746-749.

8. Strazzullo P, D’Elia L, Kandala NB, et al. (2009) Salt intake, stroke, and cardiovascular disease: meta-analysis of prospective studies. BMJ 339, b4567.

9. Ando KMH, Fujita M \& Fujita T (2010) Protective effect of dietary potassium against cardiovascular damage in salt-sensitive hypertension: possible role of its antioxidant action. Curr V asc Pharmacol 8, $56-63$.
10. Ying WZ, Aaron K, Wang PX, et al. (2009) Potassium inhibits dietary salt-induced transforming growth factor-beta production. Hypertension 54, 1159-1163.

11. Kido M, Ando K, Onozato ML, et al. (2008) Protective effect of dietary potassium against vascular injury in salt-sensitive hypertension. Hypertension 51, 225-231.

12. Matsui H, Shimosawa T, Uetake Y, et al. (2006) Protective effect of potassium against the hypertensive cardiac dysfunction: association with reactive oxygen species reduction. Hypertension 48, 225-231.

13. Chang HY, Hu YW, Yue CS, et al. (2006) Effect of potassium-enriched salt on cardiovascular mortality and medical expenses of elderly men. Am J Clin Nutr 83, 1289-1296.

14. Newby PK, Noel SE, Grant R, et al. (2010) Race and region are associated with nutrient intakes among black and white men in the United States. J Nutr 141, 296-303.

15. Yang Q, Liu T, Kuklina EV, et al. (2011) Sodium and potassium intake and mortality among US adults: prospective data from the Third National Health and Nutrition Examination Survey. Arch Intern Med 171, 1183-1191.

16. Howard VJ, Cushman M, Pulley L, et al. (2005) The reasons for geographic and racial differences in stroke study: objectives and design. Neuroepidemiology 25, 135-143.

17. Jackson R, Chambless LE, Yang K, et al. (1996) Differences between respondents and nonrespondents in a multicenter community-based study vary by gender ethnicity. The Atherosclerosis Risk in Communities (ARIC) Study Investigators. J Clin Epidemiol 49, 1441-1446.

18. Morton LM, Cahill J \& Hartge P (2006) Reporting participation in epidemiologic studies: a survey of practice. Am J Epidemiol 163, 197-203.

19. Patterson RE, Kristal AR, Tinker LF, et al. (1999) Measurement characteristics of the Women's Health Initiative food frequency questionnaire. Ann Epidemiol 9, 178-187.

20. Block G, Woods M, Potosky A, et al. (1990) Validation of a selfadministered diet history questionnaire using multiple diet records. J Clin Epidemiol 43, 1327-1335.

21. Mares-Perlman JA, Klein BE, Klein R, et al. (1993) A diet history questionnaire ranks nutrient intakes in middle-aged and older men and women similarly to multiple food records. J Nutr 123, 489-501.

22. Caan BJ, Slattery ML, Potter J, et al. (1998) Comparison of the Block and the Willett self-administered semiquantitative food frequency questionnaires with an interviewer-administered dietary history. Am J Epidemiol 148, 1137-1147.

23. Howard VJ, Kleindorfer DO, Judd SE, et al. (2011) Disparities in stroke incidence contributing to disparities in stroke mortality. Ann Neurol 69, 619-627.

24. Howard G, McClure LA, Moy CS, et al. (2011) Imputation of incident events in longitudinal cohort studies. Am J Epidemiol 174, 718-726.

25. Cook NR, Cutler JA, Obarzanek E, et al. (2007) Long term effects of dietary sodium reduction on cardiovascular disease outcomes: observational follow-up of the trials of hypertension prevention (TOHP). BMJ 334, 885-888.

26. Fukui S, Otani N, Tsuzuki N et al. (2002) Race and sex differences in the effects of dietary potassium intake on the risk of stroke [and authors' reply]. Stroke 33, 1178-1179.

27. Luft FC, Miller JZ, Grim CE, et al. (1991) Salt sensitivity and resistance of blood pressure. Age and race as factors in physiological responses. Hypertension 17, I102-I108.

28. Campese V, Parise M, Karubian F, et al. (1991) Abnormal renal hemodynamics in black salt-sensitive patients with hypertension. Hypertension 18, 805-812.

29. Calhoun DA \& Oparil S (1995) Racial differences in the pathogenesis of hypertension. Am J Med Sci 310, S91.

30. Burnier M (2008) Ethnic differences in renal handling of water and solutes in hypertension. Hypertension 52, 203-204.

31. Morris RC Jr, Sebastian A, Forman A, et al. (1999) Normotensive salt sensitivity: effects of race and dietary potassium. Hypertension 33, 18-23. 
32. Svetkey LP, Simons-Morton D, Vollmer WM, et al. (1999) Effects of dietary patterns on blood pressure: subgroup analysis of the Dietary Approaches to Stop Hypertension (DASH) randomized clinical trial. Arch Intern Med 159, 285-293.

33. Folsom AR, Parker ED \& Harnack LJ (2007) Degree of concordance with DASH diet guidelines and incidence of hypertension and fatal cardiovascular disease. Am J Hypertens 20, $225-232$.
34. Darmon N \& Drewnowski A (2008) Does social class predict diet quality? Am J Clin Nutr 87, 1107-1117.

35. Dyer A, Elliott P, Chee D, et al. (1997) Urinary biochemical markers of dietary intake in the INTERSALT study. Am J Clin Nutr 65, 1246S-1253S.

36. Toft U, Kristoffersen L, Ladelund S, et al. (2008) Relative validity of a food frequency questionnaire used in the Inter99 study. Eur J Clin Nutr 62, 1038-1046. 